Results Epithelial ASIC3 was identified in the mucosal biopsies (14 controls, 18 non-erosive reflux disease, 4 erosive oesophagitis). ASIC3 could be classified based on distribution and morphology into 4 groups; basal layer (stratum basale), stratum spinosum perinuclear, stratum spinosum membrane bound and superficial luminal layer staining. Patients who scored higher on VAS for all 6 symptoms also demonstrated higher expression of all 4 ASIC3 features studied (median $65 \%(35-87)$ vs $57 \%(34-79), p=0.018)$. In particular, for acid complaints, sleep disturbance and lower GI complaints, this association was more significant (median 64\% (46-81) vs $54 \%$ (38-69), $p=0.002)$. Nerve fibres as stained by PGP 9.5 in human oesophageal epithelium were absent.

Conclusion Presence of specific epithelial ASIC3 features in oesophageal epithelium was associated with increase in severity of common GORD symptoms. Further quantitative studies are required to study correlation of ASIC3 as an objective biomarker of symptom severity.

Competing interests None.

Keywords ASIC3, gastro-oesophageal reflux disease, Visceral Pain Hypersensitivity (VPH).

Contributors $\mathrm{Q}$ Aziz and $\mathrm{CH}$ Knowles are joint senior authors

\title{
REFERENCES
}

1. Matthews PJ, et al. Eur J Gastroenterol Hepatol 2004

2. Guarino MP, et al. Neurogastroenterol Motil 2010.

3. Bhat YM, et al. Eur J Gastroenterol Hepatol 2006.

4. Akiba Y, et al. Gut 2008.

\section{PWE-100 OESOPHAGEAL EPITHELIAL ASIC3 IS ASSOCIATED WITH INCREASE IN SEVERITY OF SYMPTOMS IN PATIENTS WITH GASTRO-OESOPHAGEAL REFLUX DISEASE (GORD)}

\author{
doi:10.1136/gut.2011.239301.363
}

\begin{abstract}
Y C Chua, ${ }^{1, *}$ A Hubball, ${ }^{1}$ M Peiris, ${ }^{1} \mathrm{~K}$ S Ng, ${ }^{1}$ S F Hughes, ${ }^{2} \mathrm{~J}$ Chinaleong, ${ }^{3}$ S Preston, ${ }^{4}$ B Patel, ${ }^{5}$ A Chaudry, ${ }^{2}$ P Levey, ${ }^{3}$ J Martin, ${ }^{3}$ O Aziz, ${ }^{1}$ C H Knowles ${ }^{1}$ Wingate Institute of Neurogastoenterology, Barts and the London School of Medicine and Dentistry, London, UK; ${ }^{2}$ Department of Surgery, Barts and the London NHS Trust, London, UK; ${ }^{3}$ Department of Pathology, Barts and the London School of Medicine and Dentistry, London, UK; ${ }^{4}$ Department of Gastroenterology, Barts and the London NHS Trust, London, UK; ${ }^{5}$ Institute of Cancer, Barts and the London School of Medicine and Dentistry, London, UK
\end{abstract}

Introduction Immunoreactive nerves and epithelial markers have been studied in the context of acid induced oesophageal hypersensitivity. Increase in immunoreactive TRPV1 nerves are associated with presence of oesophagitis ${ }^{12}$ as well as acid exposure time. ${ }^{3}$ ASIC3 was identified in epithelium of rat oesophagus and ASIC inhibitor (Amitidine) prevented oesophageal hyperaemic response in rats to $\mathrm{CO} 2$ infusion (mimicking acid). ${ }^{4}$ We aim to identify ASIC 3 in human oesophageal epithelium and to determine if it is a suitable biomarker of symptom severity in patients with gastro-oesophageal reflux disease (GORD).

Methods 36 oesophageal mucosal biopsy samples from patients (age 19-59 years and 17 females) with or without GORD undergoing gastroscopy were obtained and their clinical symptoms were assessed using visual analogue scale (VAS) adapted from validated questionnaire ReQUEST. The symptoms assessed were; general feeling, acid complaints, upper abdominal complaints, lower abdominal complaints, nausea, sleep disturbances. Samples were paraffin fixed and analysed using immunohistochemistry for PGP 9.5 and ASIC3. 